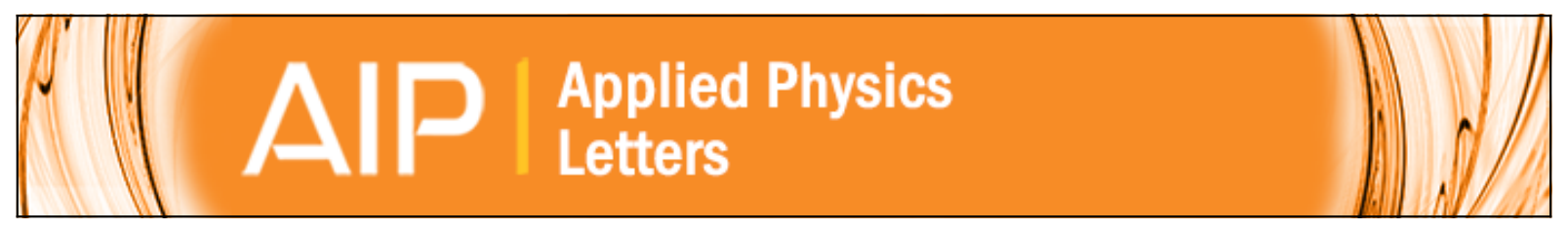

\title{
Implications of ab initio energetics on the thermodynamics of $\mathrm{Fe}-\mathrm{Cr}$ alloys
}

A. Caro, M. Caro, E. M. Lopasso, and D. A. Crowson

Citation: Applied Physics Letters 89, 121902 (2006); doi: 10.1063/1.2354445

View online: http://dx.doi.org/10.1063/1.2354445

View Table of Contents: http://scitation.aip.org/content/aip/journal/apl/89/12?ver=pdfcov

Published by the AIP Publishing

\section{Articles you may be interested in}

The Bain path of paramagnetic $\mathrm{Fe}-\mathrm{Cr}$ based alloys

J. Appl. Phys. 110, 013708 (2011); 10.1063/1.3603024

$\mathrm{Ab}$ initio spectroscopic characterization of the HNNO and ONHN radicals

J. Chem. Phys. 134, 084308 (2011); 10.1063/1.3556990

Systematic ab initio calculations on the energetics and stability of covalent $\mathrm{O} 4$

J. Chem. Phys. 120, 10084 (2004); 10.1063/1.1729923

Accurate ab initio study of the energetics of phosphorus nitride: Heat of formation, ionization potential, and electron affinity

J. Chem. Phys. 118, 8290 (2003); 10.1063/1.1565317

Gas phase ion chemistry and ab initio theoretical study of phosphine. I

J. Chem. Phys. 107, 1491 (1997); 10.1063/1.474502

\section{AlP Re-register for Table of Content Alerts}

\section{Create a profile. \\ Sign up today!}




\title{
Implications of $a b$ initio energetics on the thermodynamics of $\mathrm{Fe}-\mathrm{Cr}$ alloys
}

\author{
A. Caro a) and M. Caro \\ Chemistry and Materials Science Directorate, Lawrence Livermore National Laboratory, \\ Livermore, California 94550 \\ E. M. Lopasso \\ Centro Atmico Bariloche-Instituto Balseiro, 8400 Bariloche, Argentina \\ D. A. Crowson \\ Virginia Polytechnic Institute and State University, Blacksburg, Virginia 24061
}

(Received 5 March 2006; accepted 26 July 2006; published online 18 September 2006)

\begin{abstract}
The authors analyze the implications of the recently reported results of $a b$ initio calculations of formation energies of the $\mathrm{Fe}-\mathrm{Cr}$ alloy. The formation energies show a change in sign from negative to positive as $\mathrm{Cr}$ composition increases above $\sim 10 \%$. By developing a classic potential to evaluate the thermodynamic properties, they determine the location of the solubility limit and compare it with earlier results. A significant difference appears in a region of temperature and composition that is relevant for the nuclear applications of this alloy. Experimental results seem to confirm the validity of the location of the new solvus line. (C) 2006 American Institute of Physics.
\end{abstract}

[DOI: $10.1063 / 1.2354445]$

$\mathrm{Fe}-\mathrm{Cr}$ ferritic martensitic steels are materials of interest for nuclear applications due to their high strength at elevated temperatures and resistance to radiation damage and corrosion. The binary Fe-Cr alloy is a model system in which the basic mechanisms of radiation damage are studied with particular emphasis these days. $\mathrm{Fe}-\mathrm{Cr}$ represents a challenge for computational materials science because the magnetism in both elements combines in a complex way in the alloy. There is agreement between different sources for the phase diagram at high temperatures, see, for example, Massalsky and CALPHAD (CALculation of PHAse Diagrams) diagrams, ${ }^{1,2}$ both based on an ensemble of experimental data, the latter, in particular, based on the results of Anderson and Sunderman. ${ }^{3}$ However, at low temperature, within the ferromagnetic (FM) phase, the situation is more uncertain. The location of the solvus and the invariant line (where all three phases $\alpha, \alpha^{\prime}$, and $\sigma$ coexist) as well as the heat of formation (hof) are under discussion. Suffice it here to say that in Ref. 1 the data below $1000 \mathrm{~K}$ are drawn in dashed lines, indicating the absence of accurate experimental information.

Mirebeau et $a l^{4}$ reported experimental results on aged dilute Fe rich alloys that show the existence of short range order at $700 \mathrm{~K}$ for alloys with $\mathrm{Cr}$ content below 10 at. \%, while segregation appeared for higher concentrations. $\mathrm{Ku}-$ wano and Hamaguchi ${ }^{5}$ reported neutron irradiation results showing precipitation of $\mathrm{Cr}$ in the postirradiation annealing for $x_{\mathrm{Cr}} \geqslant 10 \%$ at $800 \mathrm{~K}$. Sagaradze et al. $^{6}$ reported electron irradiation results at the same temperature showing similar behavior. Inversion of the sign of short range order was recently confirmed by Shabashov et al. ${ }^{7}$ Finally, Mathon et al. ${ }^{8}$ studied steels with 7-10 wt \% Cr under neutron irradiation and again confirmed the separation into $\alpha$ and $\alpha^{\prime}$. From the experimental point of view it seems clear then that both the binary alloy and the steel have ordering for low $\mathrm{Cr}$ content and segregation above it, a somehow interesting behavior.

Theoretical predictions of a change in sign of the hof of the alloy started with the work of Hennion, ${ }^{9}$ who predicted it

${ }^{a)}$ Electronic mail: caro2@1lnl.gov to be at $25 \% \mathrm{Cr}$. More recently Olsson et al. ${ }^{10}$ predicted a change in sign at $6 \% \mathrm{Cr}$ and Mirzoev et al. ${ }^{11}$ analyzed in more detail the origin of the change in sign of the hof in terms of the changes in the magnetic structure. Finally, Klaver $^{12}$ offers an interpretation in terms of the antiferromagnetic alignment of the Cr substitutional solute atoms and frustration effects as $\mathrm{Cr}$ content increases. It is then a fact that the assessed phase diagrams, as those in Refs. 1 and 2, do not have the detailed information of the anomaly in the hof at low $\mathrm{Cr}$ concentration. The aim of this work is to use this information about the change in sign of the hof to determine its implications regarding the location of the solvus. We characterize this system thermodynamically and determine the modifications to the low temperature region of the phase diagram of $\mathrm{Fe}-\mathrm{Cr}$, a region of utmost interest for nuclear applications.

To go from $a b$ initio energetics to phase diagrams, the usual approach is to make approximations for the energetics of disordered configurations, like the cluster expansion method, and to assume harmonic or quasiharmonic approximations for the vibrational entropy. We instead make an approximation that consists of creating a classic potential that reproduces the energetics and several other quantities, and with it we calculate free energies with no further approximations, i.e., exact within numerical errors. The advantage of this approach is that we get a well characterized classic potential that can be later used in molecular dynamics simulations.

To determine the location of the solvus from the $a b$ initio energetics, we use our recently developed classic potential for this alloy, based on the Fe potential reported in Ref. 13 and the $\mathrm{Cr}$ potential reported in Ref. 14, that reproduces static and dynamic properties quite satisfactorily. ${ }^{15}$ The free energy calculations are then performed using a thermodynamic package that we developed for this purpose, and is reported in detail in Refs. 16-20. The Embedded Atom Model potential so developed reproduces the $a b$ initio hof reported in Ref. 10 and, as we show here, also gives an excess vibrational entropy in good agreement with 
TABLE I. Values of the Redlich-Kister expansion coefficients $L_{p}$ corresponding to the hof from Ref. 10 (in eV) and values of the coefficients $h_{i}$ in the polynomial expression of the cross potential (see text).

\begin{tabular}{ccccc}
\hline \hline$L_{0}$ & $L_{1}$ & $L_{2}$ & $L_{3}$ & $L_{4}$ \\
\hline 0.41566 & 0.0814134 & -0.0101899 & 0.267659 & -0.248269 \\
$h_{0}$ & $h_{1}$ & $h_{2}$ & $h_{3}$ & $h_{4}$ \\
\hline 1.045384 & -0.878122 & 2.130654 & -2.333204 & 0.918932 \\
\hline \hline
\end{tabular}

CALPHAD values. ${ }^{2}$ It is different from standard EAM potentials in that the cross pair term depends on both distance and local composition. We calculate Gibbs free energies for the random solid solution and liquid phases at several values of composition and temperature $G(x, T)$. Subsequently we generate a database in the THERMOCALC format and obtain the phase diagram from this software package. ${ }^{2}$ The target hof of the bcc FM solid solution is taken from Olsson et al. ${ }^{10}$ which fit with a Redlich-Kister expansion to fourth order in $(1-2 x)$ as $\Delta h=x(1-x) \Sigma_{0}^{4} L_{p}(1-2 x)^{p}$, with $x$ the $\mathrm{Cr}$ composition and parameters $L_{p}$ given in Table I.

The cross potential is given by $V_{A B}(x, r)=h(x) \frac{1}{2}\left(V_{A A}(r)\right.$ $\left.+V_{B B}(r)\right)$ with parameters in the polynomial $h(x)$ given in Table I. Figure 1 shows that the target function and the predicted value of the hof perfectly overlap. Also in Fig. 1 we show the hof of the FM phase at low $T$ as it appears in SSOL, the database of THERMOCALC. Two discrepancies are readily observable. (i) At low Cr composition, the SSOL data show no change in sign, as has been previously discussed. (ii) The hof at all compositions above $20 \%$ is greater in the ab initio result (with a maximum at $\sim 100 \mathrm{meV} /$ at.) than in SSOL (with its maximum at $\sim 60 \mathrm{meV} /$ at.). This is a point of conflict because such a difference would alter the phase diagram around equiatomic composition where the $\sigma$ phase exists, and the solubility limit of $\alpha^{\prime}$. However, three independent $a b$ initio calculations ${ }^{10-12}$ agree on a value of about $100 \mathrm{meV} /$ at. at $50 \% \mathrm{Cr}$, perhaps reflecting limitations in the magnetic structure considered or finite size effects in the $a b$ initio calculations. This point requires further investigation but does not invalidate our study of the low $\mathrm{Cr}$ content

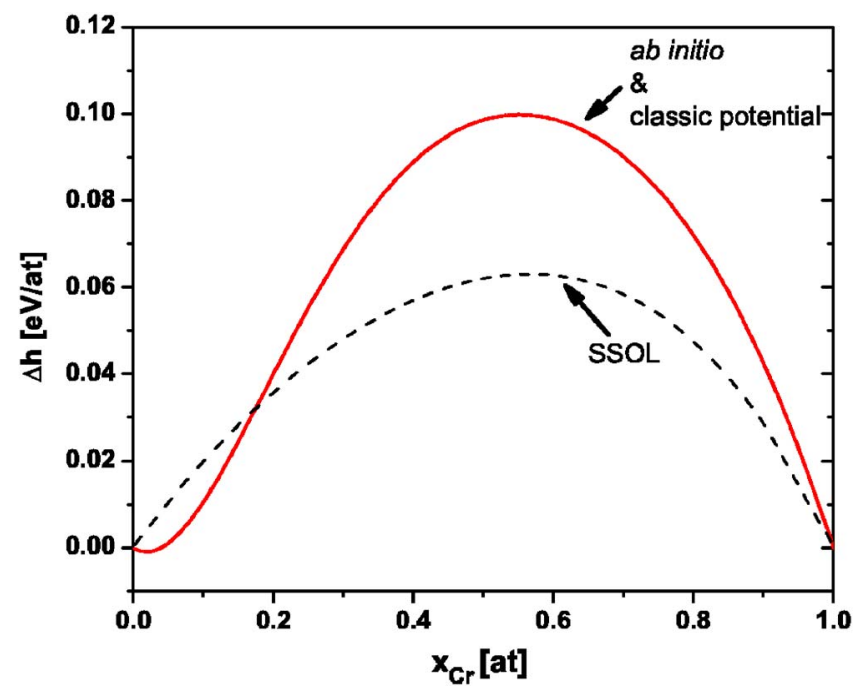

FIG. 1. (Color online) Heat of formation from Ref. 14 and predicted by the classic potential (solid line) and from SSOL (Ref. 2) (dashed line).

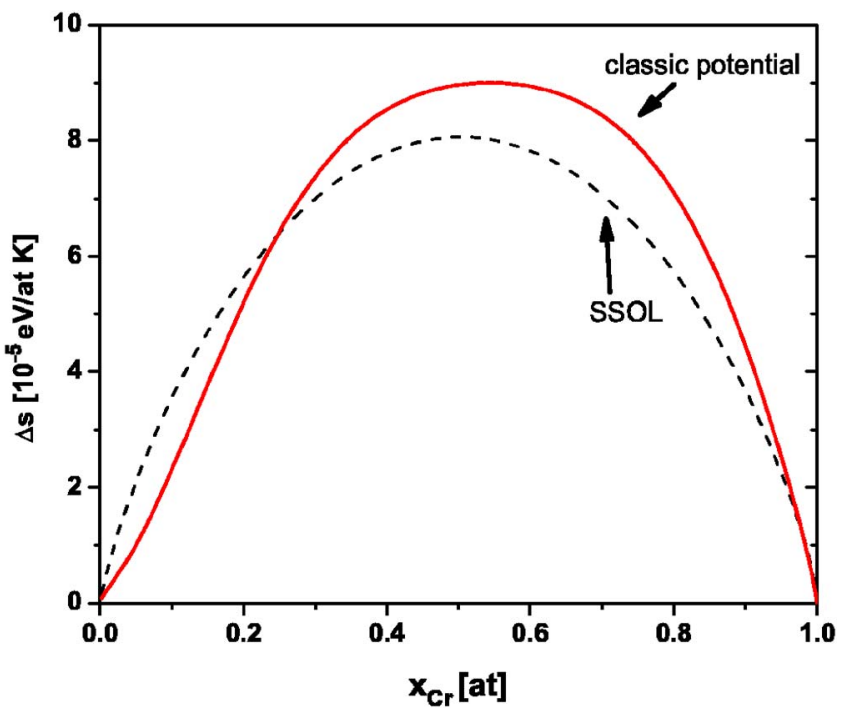

FIG. 2. (Color online) Predicted excess vibrational entropy of mixing obtained from our potential (solid line) and values of SSOL (Ref. 2) (dashed line) at low $T$ in the ferromagnetic phase.

region of the phase diagram, where the influence of the change in sign dominates the outcome.

As a test of our approach we report in Fig. 2 the excess vibrational entropy of mixing, as obtained from the free energies of both our potential and the FM phase in SSOL database. ${ }^{2}$ The agreement is good considering the fact that this quantity is not used in the development of the potential; it comes out as a prediction. We note here that despite the fact that the empirical potential does not contain any explicit degree of freedom of magnetic origin, in the FM phase that contribution to entropy is zero and therefore FM is the only phase where we should expect agreement between a classic potential and the real material.

With these free energies we get the phase diagram. Figure 3 reports the low $T$-low $x$ region of interest $0 \mathrm{~K}<T$ $<1000 \mathrm{~K}$ and $0 \%<x_{\mathrm{Cr}}<20 \%$. A continuous followed by a dotted line is our results for the solvus in the FM phase, while the dashed line is the solvus as it appears in SSOL. ${ }^{2}$ Our solvus is plotted with a solid line only for temperatures below the invariant line suggested by the experiments of Ref.

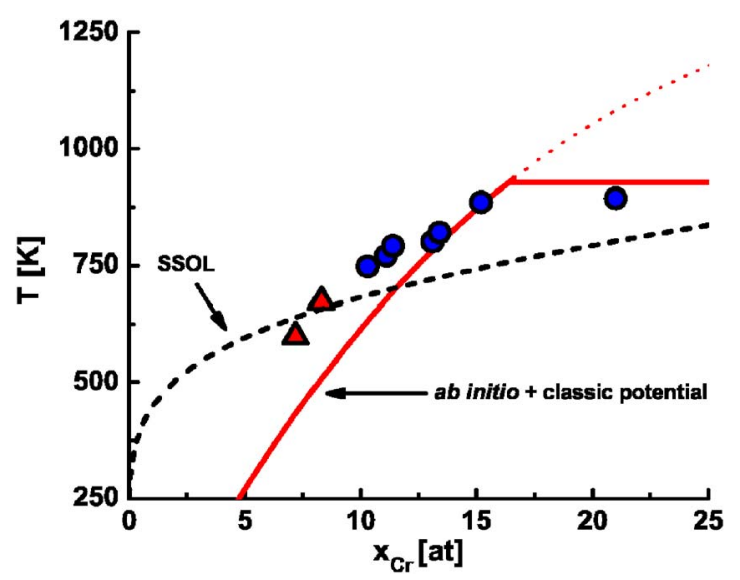

FIG. 3. (Color online) Low temperature and composition region of the phase diagram based on ab initio results from Ref. 14 generated using the $\mathrm{Fe}-\mathrm{Cr}$ empirical potential (solid and dotted lines); solvus from SSOL (Ref. 2) (dashed lines); and experimental results from Ref 5 (circles) and from Ref. 8 (triangles). 
5, that we report in the figure as a horizontal solid line; above it, the solvus is shown with a dotted line, indicating that other effects determine the diagram at those $T-x$ values, namely, the $\sigma$ phase and the magnetic transition. In Fig. 3 we have added the experimental results of Kuwano and Hamaguchi, ${ }^{5}$ and those of Mathon et $a l^{8}{ }^{8}$ The discrepancy between the SSOL solvus and the solvus implied in the ab initio results is obvious, as is the agreement between the experimental results and our prediction. The most striking difference is the fact that the solvus line, if extrapolated to zero temperature, does not cross the $x$ axis at $x=0$, as explicitly assumed in the CALPHAD treatment. This fact can easily be understood if one considers a very low $T$, where excess free energy is essentially equal to the excess heat of formation; then the solid line in Fig. 1 can be reinterpreted as a free energy curve and the common tangent construction would show that the miscibility gap at $T=0 \mathrm{~K}$ closes at $x \sim 0.04$, i.e., the location of the minimum of that curve. This fact can be considered as the main consequence of the $a b$ initio result giving a change in sign in the hof. The implications of this are as follows. Figure 3 shows how the phase diagram at low $T$ and low $x$ is modified if one considers the heat of formation predicted by $a b$ initio calculations. This diagram is calculated using a classic potential that reproduces the energetics (in particular, the change in sign at some low $\mathrm{Cr}$ concentration), and by doing the thermodynamics exactly via switching Hamiltonians and thermodynamic integration techniques. The fact that the miscibility gap closes at finite $x$ and that the maximum in the hof is higher than the SSOL value implies that the location of the solvus is significantly affected.

In summary, we observe that the change in sign of the heat of formation at about $6 \% \mathrm{Cr}$ modifies the location of the solvus in the phase diagram by making it much steeper than the SSOL value. The solubility limit above $\sim 700 \mathrm{~K}$ is much less than the value in SSOL and conversely, below $\sim 700 \mathrm{~K}$ it is much higher. In particular, at low temperature, even at $0 \mathrm{~K}$, there is a finite Cr solubility because the solvus does not go to $x_{\mathrm{Cr}}=0$ at $T=0$ but to $x_{\mathrm{Cr}} \sim 0.04$. As we pointed out above, the finite $\mathrm{Cr}$ solubility at $0 \mathrm{~K}$ is a result that is not affected by the controversy regarding the value of the hof around equiatomic composition. However, how steep the solvus is at both ends of the diagram strongly depends on that value. The solubility of $\mathrm{Fe}$ in the $\alpha^{\prime}$ phase, as we predict it, is too low compared to experiments ${ }^{5}$ and SSOL, a result also related to the high hof predicted $a b$ initio.

This work was performed under the auspices of the U.S. Department of Energy by the University of California, Lawrence Livermore National Laboratory under Contract No. W-7405-Eng-48, with support from the Laboratory Directed Research and Development Program.

${ }^{1}$ T. Massalski, in Binary Alloy Phase Diagrams, edited by William W. Scott, Jr. (American Society for Metals, Metals Park, OH, 1986), Vol. 1, p. 822.

${ }^{2}$ N. Saunders and A. P. Miodownik, in CALPHAD: A Comprehensive Guide, edited by R. W. Cahn (Pergamon, Oxford, 1998).

${ }^{3}$ J. O. Anderson and R. Sunderman, CALPHAD: Comput. Coupling Phase Diagrams Thermochem. 11, 83 (1987).

${ }^{4}$ I. Mirebeau, M. Hennion, and G. Parette, Phys. Rev. Lett. 53, 687 (1984).

${ }^{5}$ H. Kuwano and Y. Hamaguchi, J. Nucl. Mater. 155, 1071 (1988).

${ }^{6}$ V. V. Sagaradze, I. I. Kositsyna, V. L. Arbuzov, V. A. Shabashov, and Y. I. Filippov, Phys. Met. Metallogr. 92, 508 (2001).

${ }^{7}$ V. A. Shabashov, A. L. Nikolaev, A. G. Mukoseev, V. V. Sagaradze, and N. P. Filippova, Bull. Russ. Acad. Sci. Phys. 65, 1094 (2001).

${ }^{8}$ M. H. Mathon, Y. de Carlan, G. Geoffroy, X. Averty, and A. Alamo, J. Nucl. Mater. 312, 236 (2003).

${ }^{9}$ M. Henion, J. Phys. F: Met. Phys. 13, 2351 (1983).

${ }^{10}$ P. Olsson, I. A. Abrikosov, L. Vitos, and J. Wallenius, J. Nucl. Mater. 321, 84 (2003).

${ }^{11}$ A. A. Mirzoev, M. M. Yalalov, and D. A. Mirzaev, Phys. Met. Metallogr. 97, 336 (2003).

${ }^{12} \mathrm{P}$. Klaver (private comunication).

${ }^{13}$ M. I. Mendelev, S. Han, D. J. Srolovitz, G. J. Ackland, D. Y. Sun, and M. Asta, Philos. Mag. 83, 3977 (2003).

${ }^{14}$ J. Wallenius, P. Olsson, C. Lagerstedt, N. Sandberg, R. Chakarova, and V. Pontikis, Phys. Rev. B 69, 094103 (2004).

${ }^{15}$ A. Caro, D. A. Crowson, and M. Caro, Phys. Rev. Lett. 95, 075702 (2005).

${ }^{16}$ E. O. Arregui, M. Caro, and A. Caro, Phys. Rev. B 66, 054201 (2002).

${ }^{17}$ A. Caro, P. E. A. Turchi, M. Caro, and E. M. Lopasso, J. Nucl. Mater. 336, 233 (2005).

${ }^{18}$ A. Caro, M. Caro, E. M. Lopasso, P. E. A. Turchi, and D. Farkas, J. Nucl. Mater. 349, 317 (2006).

${ }^{19}$ E. M. Lopasso, M. Caro, A. Caro, and P. E. A. Turchi, Phys. Rev. B 68, $214205(2003)$

${ }^{20}$ A. Caro, M. Caro, E. M. Lopasso, P. E. A. Turchi, and D. Farkas, J. Nucl. Mater. 336, 233 (2004). 